\title{
Metastasis to Submandibular Salivary Gland in Oral Carcinoma
}

\author{
Raana Akbar ${ }^{1 *}$, Shakil Aqil', Saeed Akhtar ${ }^{1}$ and Ahmed Nawaz ${ }^{1}$ \\ ${ }^{1}$ Department of Otorhinolaryngology Head and Neck Surgery, Liaquat National Hospital and Medical College, Karachi, Pakistan
}

\begin{abstract}
Objective: To determine the frequency of involvement of the submandibular gland as a metastatic site in squamous cell carcinoma of oral cavity, mechanism of its involvement and factors associated with it.

Methods: We reviewed the medical and pathologic records of patients who underwent neck dissection (unilateral or bilateral) for oral cavity squamous cell carcinomas (SCC) from January 2009 to July 2017 at the Liaquat National Hospital in Karachi. Cases of histopathologically confirmed SCC of oral cavity and cases of primary tumor in which excision of the submandibular gland (SMG) was done along with neck dissection were included. Those cases with previous surgery for oral cancer and those with previous chemoradiotherapy were excluded.

Results: Median age at presentation was 49.50 years (IQR= 42-58 years). Males were more commonly affected ( $n=167,79.5 \%)$. The most frequently involved site was buccal mucosa $(n=110,52.4 \%)$. Out of 210 neck dissections, SMG was involved by the tumor metastasis in only $8(3.8 \%)$ of the cases. Most common method of involvement was direct invasion by the primary tumor $(n=5$, $62.5 \%)$. Floor of mouth $(p<0.001)$ and level 1 lymph node involvement $(p<0.001)$ were significantly associated with SMG involvement.

Conclusion: SMG is the major source of unstimulated saliva. Saliva plays an important role in maintaining oral hygiene and health. Especially in patients of oral cavity SCC who receive post-operative radiotherapy its removal can aggravate xerostomia which can lead to severe discomfort. In this study there was no involvement of SMG in cases in which floor of mouth was not involved and in cases with clinically negative neck. So we concluded that efforts should be made to preserve SMG in these cases to reduce postoperative morbidity associated with its removal.
\end{abstract}

Keywords: Oral cavity squamous cell carcinoma, submandibular gland metastasis, neck dissection, Xerostomia.

\section{INTRODUCTION}

Oral cancer is one of the most common types of cancer in head and neck (38\%). 95\% of them are squamous cell carcinomas (SCC). It is one of the 10th most common malignancies worldwide while in Karachi it is 2nd most common cancer [1]. SCC has a tendency to metastasize to the neck. In oral cancer it is in $30 \%$ of T1 and T2 lesions and about $60 \%$ in T3 and T4 lesions [2]. Surgical treatment includes wide excision of primary lesion with or without neck dissection.

Neck dissection has proved to be an essential procedure in the treatment of head and neck cancer. It is a standard practice to include SMG when level lb lymph nodes; present in submandibular triangle; are removed regardless of type of neck dissection done [3]. However, SMG has an important function to serve. It is responsible for $60-70 \%$ of unstimulated basal flow of saliva. Removing even one of the SMG has negative impact on salivary flow. Saliva is not only important for lubrication of oral cavity but is also vital for mucosal immunity and preparation of food bolus during mastication [4].

Since the first description of neck dissection was made by Crile in 1906, many refinements and modifications have been made and the trend is shifting from radical clearance to more selective and functional procedures [4].

*Corresponding Author: Raana Akbar, Department of Otorhinolaryngology Head and Neck Surgery, Liaquat National Hospital and Medical College, Karachi, Pakistan; Email: dr.raana@yahoo.com

Received: June 20, 2020; Revised: July 12, 2020; Accepted: July 13, 2020 DOI: https://doi.org/10.37184/lntbj.2708-7808.2.5
In light of more conservative and individually tailored surgery, the question arises whether it is necessary to remove SMG in neck dissection. It is believed that level $1 \mathrm{~b}$ clearance is not achieved without excision of SMG and also because of its involvement by oral cavity cancer.

In a study done by Dhiwakar et al. in 2009, 33 neck dissections along with SMG excision was done. Average of 1 to 15 lymph nodes was recovered form level $1 b$ in a 3 step procedure. In first step all lymph nodes above the submandibular gland were removed, then in second step SMG was removed and in the last step all fibro fatty tissue from the bed of gland was removed and histopathological analysis was done. It was found that all of the lymph nodes were recovered before the removal of SMG. There were no lymph nodes found with SMG and at its bed and there were no intra glandular lymph nodes as well [5].

Other studies have also shown that unlike the parotid gland SMG does not contain any internal lymphoid tissue, and have reported the prevalence of its involvement by head and neck cancer from 0.9 to $5.3 \%$ [6].

There are 3 ways by which SMG is involved by the oral cavity tumors. These are direct invasion by the tumor (which is most common), direct invasion by periglandular lymph nodes and by SMG metastasis. Basaran et al. studied histopathologic records of 236 patients and found that only 13 cases $(4 \%)$ had SMG involvement. Tumor sites most likely to involve SMG are lower alveolus and floor of mouth (FOM) because of their close proximity 
to the SMG. They recommended that SMG should be preserved in all cases except in those that have direct invasion/contact by primary tumor or by metastatic lymphadenopathy [7].

In this study we wanted to find out the frequency of involvement of SMG as a metastatic site in oral cavity squamous cell carcinoma (OCSCC) and also to find out which factors are likely to predict SMG metastasis. The factors studied were tumor size, site and grade; level 1 lymph node, floor of mouth (FOM) and bone involvement. Studying FOM involvement is important because mylohyoid musle which comprises the major part of FOM also forms the bed of SMG. We also wanted to find out the mechanism of involvement of SMG. This will help us in identifying those cases of OCSCC in which SMG is not involved and hence, can be preserved during neck dissection. The objective is to determine the frequency of involvement of submandibular gland, mechanism of its involvement and factors associated with it.

\section{MATERIALS AND METHODS}

We reviewed the medical and pathologic charts of 210 patients who underwent surgery for OCSCC at ENT Head and Neck Surgery Department of the Liaquat National Hospital between the years 2009 and 2017. Cases of histopathologically confirmed SCC of oral cavity and cases of primary tumor in which excision of the SMG was done along with neck dissection were included. Those cases with previous surgery for oral cancer and those with previous chemo-radiotherapy were excluded.
Data was analyzed using SPSS (version 20.0). Categorical variables were described as frequencies and percentages. Quantitative variable age was described as median with interqaurtile range (IQR) after assessing assumption of normality with Shapiro-wilk test. To find out the association between tumor related factors and SMG involvement, chi-square or Fisher's exact test was applied as appropriate. P-value of $<0.05$ was considered as statistically significant.

\section{RESULTS}

The median age at presentation was 49.50 years (IQR= $42-58$ years), of which 167 (79.5\%) were males and 43 $(20.5 \%)$ were females. Buccal mucosa was the most common site of involvement $(n=110,52.4)$ followed by tongue $(n=66,31.4 \%)$, lower alveolus $(n=17,8.1 \%)$, hard palate $(n=6,2.9 \%)$, retromolar trigone $(n=5,2.4 \%)$, lip $(n=5,2.9 \%)$. Most of the patients presented with tumor size of $2-4 \mathrm{~cm}(n=112,53.3 \%)$ whereas nearly one-fourth of the patients also presented with tumor size of $\leq 2 \mathrm{~cm}(n=46,21.9 \%)$ and $>4 \mathrm{~cm}(n=52,24.8)$. More than half of the patients had moderately differentiated tumor $(n=155,73.8 \%)$ and few also presented with well differentiated $(n=21,10 \%)$ and poorly differentiated $(n=34,16.2 \%)$ tumor grade. In majority of the cases, bone involvement was not observed ( $n=169,80.5 \%)$. More than quarter of the patients had level 1 neck node involvement ( $n=59,28.1 \%)$.

Very few patients presented with SMG involvement $(n=8,3.8 \%)$. In $5(62.5 \%)$ cases SMG was involved due

Table 1: Association of various tumor related factors with SMG involvement.

\begin{tabular}{|c|c|c|c|}
\hline Variables & $\begin{array}{c}\text { With Submandibular } \\
\text { Invovlement } \\
\mathrm{n}(\%)\end{array}$ & $\begin{array}{c}\text { Without Submandibular } \\
\text { Invovlement } \\
\mathrm{n}(\%)\end{array}$ & p-value \\
\hline \multicolumn{4}{|l|}{ Tumor Grade } \\
\hline Moderately differentiated & $7(4.5)$ & $148(95.5)$ & \multirow[t]{2}{*}{+1.00} \\
\hline Poorly differentiated & $1(2.9)$ & $33(97.1)$ & \\
\hline \multicolumn{4}{|l|}{ Tumor Size } \\
\hline$>4 \mathrm{~cm}$ & $4(7.7)$ & $48(92.3)$ & †0.307 \\
\hline \multicolumn{4}{|l|}{ Tumor Site } \\
\hline Buccal mucosa & $5(4.5)$ & 105(95.5) & +0.724 \\
\hline Tongue & $2(3)$ & $64(97)$ & +1.00 \\
\hline Lower alveolus & $1(5.9)$ & $16(94.1)$ & +1.00 \\
\hline Floor of mouth & $5(20)$ & $20(80)$ & $t^{\star *}<0.001$ \\
\hline \multicolumn{4}{|l|}{ Level 1 LN Involved } \\
\hline Yes & $8(13.6)$ & $51(86.4)$ & \multirow{2}{*}{${ }^{* *}<0.001$} \\
\hline No & $0(0)$ & $151(100)$ & \\
\hline \multicolumn{4}{|l|}{ Bone Involvement } \\
\hline Yes & $3(7.3)$ & $38(92.7)$ & \multirow{2}{*}{ t0.189 } \\
\hline No & $5(3)$ & 164(97) & \\
\hline
\end{tabular}

† Fisher's exact test was applied, ** Significant at $P<0.01$ 
to direct invasion from the primary tumor. $2(25 \%)$ cases were those in which SMG was involved through the metastatic periglandular lymph node. There was only 1 $(12.5 \%)$ gland in which neither there was direct tumor invasion nor through the periglandular metastatic lymph node and it was labeled as SMG metastasis.

We studied association of various tumor related factors with SMG involvement. Table 1 shows association of various tumor related factors with SMG involvement.

Different tumor sites including buccal mucosa $(p=0.724)$, tongue $(p=1.00)$, lower alveoluos $(p=1.00)$, hard palate $(p=1.00)$, lip (1.00) upper alveoluos were not significantly different among patients with and without SMG involvement. However, only floor of mouth was significantly associated with SMG involvement $(p<0.001)$. There were $25(11.9 \%)$ patients with tumor present at FOM and out of them, $5(20 \%)$ were those who have involvement of SMG. While in remaining 185 $(88.1 \%)$ cases in which there was no FOM involvement, only $3(1.6 \%)$ patients had involvement of SMG. Level 1 lymph node involvement was also associated with SMG involvement $(p<0.001)$. Among $59(28 \%)$ patients with level 1 lymph node involvement, 8 (13.6\%) had SMG involvement while those patients without level 1 lymph node involvement none had SMG involvement.

\section{DISCUSSION}

Saliva has many important functions such as lubrication, tooth mineralization, taste perception and has antimicrobial activity. Most of these protective functions of saliva is due to the unstimulated saliva and SMG produces 70 to $80 \%$ of this unstimulated saliva. Removal of even 1 SMG leads to significant decrease in flow of unstimulated saliva leading to xerostomia [8]. In neck dissection for oral cavity SCC SMG is excised due to two reasons; due to clearance of level $1 \mathrm{~b}$ and due to SMG invasion by tumor or metastatic lymph node. Level $1 \mathrm{~b}$ lymph node dissection can be carried out easily especially in node negative neck because there are no nodes at the bed of the gland [9]. Metastasis to the SMG is rare and it is through the hematogenous route especially in breast, lung and genitourinary cancers [10]. For OCSCC the possibility of SMG involvement is very low because it does not have any intraglandular lymphatic channels. Chen reported 1 case of metastasis. Vaidva reported 2 cases; one had tongue as primary site and other had palate. He observed that in both cases neck lymph nodes were not involved by metastasis [11, 12].

Various studies done internationally also showed that SMG involvement is $<5 \%$ in OCSCC. Spiegel in his study recommended that bilateral SMG should not be excised and decision regarding excision of gland should be made per operatively on the basis of proximity/ adherence of primary tumor or tumor metastasis to the gland $[13,14]$.
In our study there was only 1 case of SMG metastasis which neither had direct tumor invasion nor through the periglandular metastatic lymph node. In this particular case primary site was of buccal mucosa measuring $3 \times 2.5 \mathrm{~cm}$ with moderate to poor differentiation and perineural invasion. FOM was not involved. Neck dissection specimen showed one lymph node positive at level 1 but without perinodal extension. In other studies also it was found that SMG is only rarely involved by tumor because it lacks intraglandular lymphatic channels. Hematogenous route could be the reason of SMG involvement from tumors outside head and neck region [15].

So, in advanced oral cavity tumors; in which FOM is not involved; effort should be made to preserve SMG because post-operative adjuvant radiation therapy (RT) also damages salivary glands and aggravates xerostomia [16]. Parotid glands, which have an affinity to damage by RT, are affected rapidly and irreversibly. SMG is less sensitive to radiation so it is less affected. Transfer of SMG to submetal region during neck dissection is also possible, this will further reduce postoperative xerostomia accelerated by RT $[17,18]$.

\section{CONCLUSION}

On the basis of our study we recommend that SMG should not be excised in cases in which FOM is not involved by the primary tumor and in cases in which level 1 lymph nodes are not involved. However, effect of preservation of SMG on loco regional control of disease is yet to be studied.

\section{CONFLICT OF INTEREST}

The authors declare no conflict of interest.

\section{ACKNOWLEDGEMENTS}

Declared none.

\section{REFERENCES}

1. Bhurgri $\mathrm{Y}$, Bhurgri $\mathrm{A}$, Hassan $\mathrm{SH}$, et al. Cancer incidence in Karachi, Pakistan: first results from Karachi cancer registry. Int J Cancer 2000; 85(3): 325-9.

2. Okada $Y$, Mataga I, Katagiri $M$, et al. An analysis of cervical lymph nodes metastasis in oral squamous cell carcinoma: relationship between grade of histopathological malignancy and lymph nodes metastasis. Int J Oral Max Surg 2003; 32(3): 284-8.

3. Rani P, Bhardwaj Y, Dass PK, et al. Neck dissection for oral squamous cell carcinoma: our experience and a review of the literature. J Korean Assoc Oral Maxillofac Surg 2015; 41(6): 299305.

4. Byeon HK, Lim YC, Koo BS, et al. Metastasis to the submandibular gland in oral cavity squamous cell carcinomas: pathologic analysis. Acta Otolaryngol 2009; 129(1): 96-100.

5. Dhiwakar M, Ronen O, Malone J, et al. Feasibility of submandibular gland preservation in neck dissection: a prospective anatomicpathologic study. Head Neck 2011; 33(5): 603-9.

6. Howard BE, Hinni ML, Nagel TH, et al. Submandibular gland preservation during concurrent neck dissection and transoral surgery for oropharyngeal squamous cell carcinoma. Otolaryngol Head Neck Surg 2014; 150(4): 587-93. 
7. Basaran B, Ulusan M, Orhan KS, et al. Is it necessary to remove submandibular glands in squamous cell carcinomas of the oral cavity? Acta Otolaryngol 2013; 33(2): 88.

8. Jacob RF, Weber RS, King GE. Whole salivary flow rates following submandibular gland resection. Head Neck 1996; 18: 242-7.

9. Takes RP, Robbins KT, Woolgar JA, et al. Questionable necessity to remove the submandibular gland in neck dissection. Head Neck 2011; 33: 743-5.

10. Ebrahim AK, Loock JW, Afrogheh A, et al. Is it oncologically safe to leave the ipsilateral submandibular gland during neck dissection for head and neck squamous cell carcinoma? J Laryngol Otol 2011; 125(8): 837.

11. Chen TC, Lou PJ, Ko JY, et al. Feasibility of preservation of the submandibular gland during neck dissection in patients with earlystage oral cancer. Ann Surg Oncol 2011; 18(2): 497-504.

12. Vaidya AM, Vaidya AM, Petruzellie GJ, et al. Isolated submandibular gland metastasis from oral cavity squamous cell carcinoma. Am J Otolaryngol 1999; 20: 172-5.
13. Spiegel JH, Brys AK, Bhakti A, et al. Metastasis to the submandibular gland in head and neck carcinomas. Head Neck 2004; 12: 1064-8.

14. Jha N, Seikaly $H$, Harris J, et al. Prevention of radiation induced xerostomia by surgical transfer of submandibular salivary gland into the submental space. Radiother Oncol 2003; 66: 283-9.

15. Razfar A, Walvekar RR, Melkane A, et al. Incidence and patterns of regional metastasis in early oral squamous cell cancers: feasibility of submandibular gland preservation. Head Neck 2009; 10: 161923

16. Chambers MS, Garden AS, Kies MS, et al. Radiation-induced xerostomia in patients with head and neck cancer: pathogenesis, impact on quality of life, and management. Head Neck 2004; 26 : 796-807.

17. Chen TC, Lo WC, Ko JY, et al. Rare involvement of submandibular gland by oral squamous cell carcinoma. Head Neck 2009; 31: 87781.

18. Seikaly $H$, Jha N, McGaw T, et al. Submandibular gland transfer: a new method of preventing radiation induced xerostomia. Laryngoscope 2001; 111: 347-52. 